\title{
PROTON ACCELERATION IN PLASMA WAVES PRODUCED BY BACKWARD RAMAN SCATTERING
}

\author{
A. Ogata, AdSM, Hiroshima University, Kagamiyama, Higashi-Hiroshima, 739-8526, JAPAN \\ T. Katsouleas, University of Southern California, Los Angeles, CA 90089-0484 USA
}

\section{Abstract}

This paper proposes a new method of proton acceleration. A slow plasma wave produced by backward Raman scattering captures slow protons injected. A density gradient of a plasma enables the wave phase velocity to increase as the proton test beams are accelerated. A proof-of-principle experiment is proposed using a $\mathrm{T}^{3}$ laser and test beams from a Van de Graaff accelerator, in which a 500mJ laser will give acceleration gain of $\sim 50 \mathrm{MeV}$ in a distance of $500 \mu \mathrm{m}$.

\section{INTRODUCTION}

This paper proposes a proton acceleration using plasma waves[1], based on the same principle which has successfully accelerated electrons with a large acceleration gradient. It uses the electric field of a plasma wave excited by a laser. The laser is scattered into either forward or backward directions in a plasma. The forward scattering excites a plasma wave whose phase velocity is almost equal to the group velocity of the pump laser. This fast plasma wave has been used in electron acceleration. This method is called self-modulated laser wakefield acceleration, because the laser pulse is modulated at the plasma frequency by the forward instability. To the contrary, the backward scattering excites a slow plasma wave, and it is this slow wave that we are going to use for proton acceleration.

Relativistic electrons have light velocity, so they can be synchronous with the relativistic plasma wave whatever energy they have. Protons below $1 \mathrm{GeV}$, however, increases their velocity as they are accelerated. The technical difficulty of the proton acceleration using a plasma wave is to give it increasing phase velocity synchronous with the accelerated proton velocity. In this proposal, the synchronization is enabled by the density distribution of the plasma.

This paper consists of five sections. Following this introductory section, the second section describes the phase velocity of the plasma wave that can trap the protons. The third section briefly describes backward Raman scattering. The fourth section proposes acceleration in a plasma with tapered density, in which the phase velocity of the plasma wave increases as the test beam is accelerated. A proofof-principle experiment is also proposed. The last section gives discussion and conclusions.

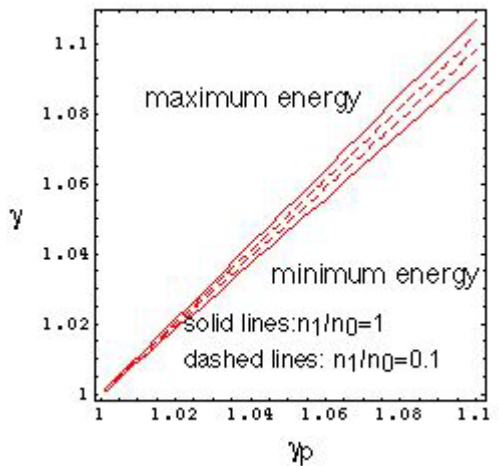

Figure 1: Maximum and minimum proton energies that can be trapped by a plasma wave with $\gamma_{p}$. Solid lines: $n_{1} / n_{0}=$ 1, broken lines: $n_{1} / n_{0}=0.1$.

\section{TRAPPING OF PROTONS IN A PLASMA WAVE}

The motion of protons in a plasma wave is described by a pair of equations:

$$
\begin{aligned}
& d \gamma / d z=-\left(e E / m_{p} c^{2}\right) \sin \phi, \\
& d \phi / d z=k_{p}\left(1 / \beta_{p}-1 / \beta\right),
\end{aligned}
$$

where $E$ is the field of the plasma wave, $m_{p}$ is the proton mass, $\beta$ and $\gamma$ are those of the protons and $\beta_{p}$ is that of the plasma wave. They mean that the wave phase velocity $\beta_{p}$ should be equal to the particle velocity $\beta$, otherwise the energy gain cannot be constant.

However, the protons can remain to be trapped by the wave even if the condition $\beta_{p}=\beta$ is not strictly satisfied, as far as $\sin \phi<0$ in eq. (1). This condition of trapping is equivalent to that the kinetic energy of protons is smaller than the wave potential $\Phi[2]$. We use prime symbols to denote variables in the wave frame. The condition then becomes

$$
\left(\gamma^{\prime}-1\right) m_{p} c^{2} \leq e \Phi^{\prime}
$$

so the boundary energy of the protons that can be trapped is $\gamma^{\prime} m_{p} c^{2}=e \Phi^{\prime}+m_{p} c^{2}$. In the laboratory frame we have two boundary energies,

$$
\gamma_{ \pm}=\gamma_{p} \gamma^{\prime}\left(1 \pm \beta_{p} \beta^{\prime}\right)
$$

Using $e E=e k_{p}^{\prime} \Phi^{\prime}=e k_{p} \Phi$ and $e E=\left(n_{1} / n_{0}\right) m_{e} \omega_{p} v_{p}$ we have $\gamma^{\prime}=\left(n_{1} / n_{0}\right)\left(m_{e} / m_{p}\right) \gamma_{p} \beta_{p}^{2}+1$, where $n_{1}$ is the electron density in the wave and $n_{0}$ is the background 


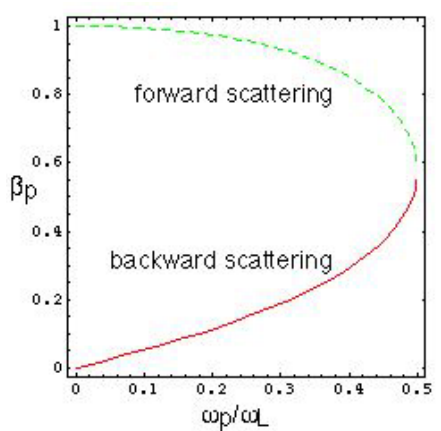

Figure 2: Dependence of phase velocity of the plasma wave produced in the Raman scattering on $\omega_{p} / \omega_{L}$.

electron density. Inserting this into eq.(3), we have the relation between $\gamma$ and $\gamma_{p}$, which is depicted in Fig.1. This figure gives the energy range of protons that are trapped by a plasma wave at specific $\gamma_{p}$. This width is $\sim \pm 7 \%$ at $\gamma_{p}=1.1$ and $n_{1} / n_{0}=1$, and decreases as $\gamma_{p}$ and $n_{1} / n_{0}=1$ decrease. We have to tune the phase velocity of the plasma wave within this range in order to perform the successful acceleration.

\section{BACKWARD RAMAN SCATTERING}

In the forward Raman scattering, both the plasma wave (denoted by suffix 'p' hereafter) and the scattered radiation with suffix ' $F$ ' propagate in the same direction as the pump radiation (denoted by suffix ' $L$ '). In the backward scattering, the scattered radiation with suffix ' $\mathrm{B}$ ' propagates in the opposite direction, though the plasma wave has the same direction as the pump radiation. The dispersion relations of the electromagnetic waves in a cold plasma are given by $\omega_{x}^{2}=\omega_{p}^{2}+c^{2} k_{x}^{2}$, where $x=L, F$ or $B$. The conditions for resonance are $\omega_{L}=\omega_{p}+\omega_{x}, k_{L}=k_{p}+k_{F}$, and $k_{L}=k_{p}-k_{B}$, where $x=F$ or $B$. They give the phase velocity of the plasma wave $v_{p}=c \beta_{p}$, where

$$
\beta_{p}=\frac{\omega_{p}}{\omega_{L}} \frac{1}{\sqrt{1-\left(\omega_{p} / \omega_{L}\right)^{2}} \mp \sqrt{1-\left(2 \omega_{p} / \omega_{L}\right)}},
$$

with - and + signs corresponding to forward and backward scatterings, respectively. It is shown in Fig.2 as a function of $\omega_{p} / \omega_{L}$. As is clear in the figure, the phase velocity of the plasma wave produced by the backward scattering is the increasing function of the plasma density. The phase velocity curves of the forward and backward scatteringproduced plasmas merge at the point where $\omega_{L}=2 \omega_{p}$, at which $\beta_{p}=1 / \sqrt{3}$.

The backward Raman scattering had been studied vigorously in the 1970's in connection with laser fusion. We summarize here the main features obtained then [3].

Growth rate of the backward Raman scattering is given by $\gamma=\left(v_{q} / c\right)^{2}\left(\omega_{L} \omega_{p}\right)^{1 / 2}$. Landau damping suppresses this scattering, unless [3]

$$
\omega_{p} / \omega_{L}>2 v_{t h} / c,
$$

where $v_{t h}=\left(k_{B} T_{e} / m_{e}\right)^{1 / 2}$. This requirement is most difficult to fulfill at beam injection. Approximating $k_{p}=2 k_{L}$ and $\omega_{L}=c k_{L}$, we have $\omega_{p} / \omega_{L}=2 \beta_{p}$. The $\beta$ value of the injected protons should be equal to the $\beta_{p}$ value of the plasma wave. The condition of eq.(5) then becomes $\beta>v_{t h} / c$. This restriction limits the lowest-possible injection energy. Electron temperatures $10 \mathrm{eV}, 100 \mathrm{eV}, 1 \mathrm{keV}$ $\ldots$ requires the injection $\beta$ of $0.006,0.02,0.06, \ldots$, respectively.

The plasma wave decay can be slower than the growth of the backward instability. However, once the pump laser fades out, the plasma wave decays so quickly because of the Landau damping that we cannot expect a short laser pulse to leave a wake which survives until a slow proton beam arrives. In other words, the laser pulsewidth has to be longer than the acceleration time. This situation differs from the case of electron acceleration where "wakefield acceleration" is possible.

The electric field associated with a fast plasma wave is approximately $e E=\left(n_{1} / n_{0}\right) m_{e} \omega_{p} c$. This cannot be applied to our case of a slow plasma wave. We have instead

$$
e E=\left(n_{1} / n_{0}\right) m_{e} \omega_{p} v_{p} .
$$

The Manley-Rowe relation gives the maximum-possible $n_{1} / n_{0}$ value as a function of the electron quiver velocity $v_{q}$ in the laser field[4]:

$$
\left(n_{1} / n_{0}\right)^{2} \sim\left(\omega_{L} / \omega_{p}\right)\left(2 v_{q}^{2} / \gamma v_{t h}^{2}\right),
$$

where $\gamma$ is the specific heat ratio. In one-dimensional model we have $\gamma=3$.

In this proposal, we use a plasma with tapered density. In such an inhomogeneous plasma, the resonant condition can hold only locally. The propagation of the plasma wave out of this resonant region then provides an additional threshold, which is [3],

$$
\left(v_{q} / c\right)^{2} k_{L} L>1, \quad\left(2 \omega_{p}<\omega_{L}\right),
$$

where $L=[(1 / n)(d n / d s)]^{-1}$ is a length characterizing the density gradient, with $s$, the distance along the density gradient.

The laser-driven instability growth has to balance the plasma wave decay. It remains an open question whether such a balance is achievable. One solution to control this instability is the use of a second laser which acts as a seed for the instability[1]. This seed laser should be frequencyshifted by $\omega_{p}$ from the pump laser and injected from the counter direction of the pump laser. In other words, the seed laser should have the same frequency and direction of the backscattered radiation. This is a beatwave acceleration in which the beat frequency of pump and seed lasers is equal to the plasma frequency.

\section{TAPERED DENSITY METHOD AND PROOF-OF-PRINCIPLE EXPERIMENT}

We now try to carry out a simple calculation, with a proof-of-principle experiment in mind. We assume to 

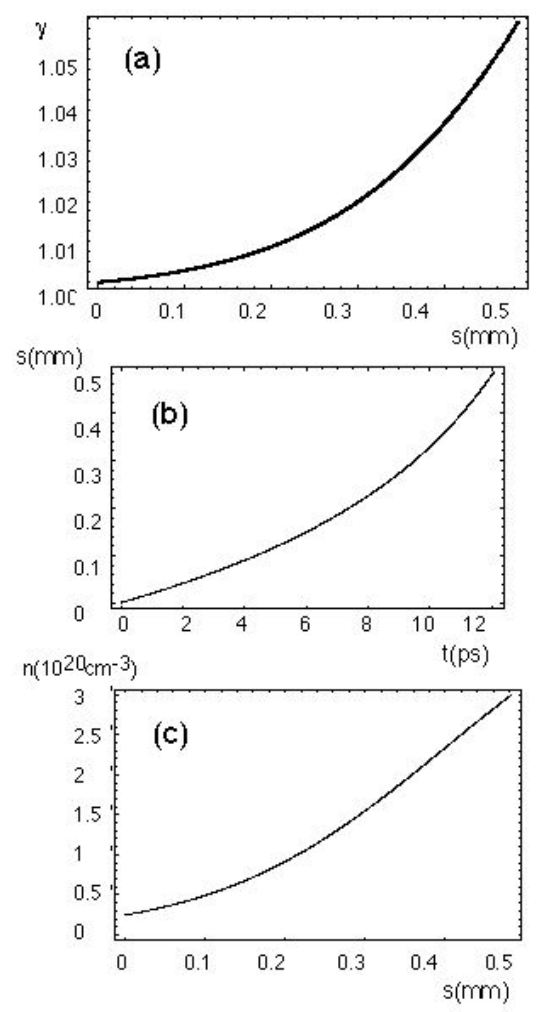

Figure 3: Results of the calculation. (a) beam energy $(\gamma)$ vs. distance, (b) propagation distance vs time, (c) required plasma density vs distance.

use $2.5 \mathrm{MeV}$ proton beams $(\beta=0.078, \gamma=1.00267)$ from a Van de Graaff of Hiroshima University, and a Ti:sapphire laser with $\lambda_{L}=800 \mathrm{~nm}$. We set the final energy as $50 \mathrm{MeV}(\beta=0.314, \gamma=1.0533)$. We assume $n_{1} / n_{0}=1 / 2$ to have the acceleration gradient as $e E(s)=m_{e} c \beta_{p}(s) \omega_{p}(s) / 2$. A more realistic $n_{1} / n_{0}$ value should be determined by PIC simulations.

The calculation is based on eq.(1): The second equation is used to derive the $\beta_{p}$ which makes $d \phi / d t=0$ for given $\beta$ by the first equation. The plasma density to give this $\beta_{p}$ is the solution of eq.(4).

Figure 3 shows results of the calculation. Figure 3 (a) and (b) show that the energy gain of $\sim 50 \mathrm{MeV}$ is attained in the acceleration time of $\sim 12 \mathrm{ps}$ and acceleration distance of $\sim 0.5 \mathrm{~mm}$. Figure 3 (c) shows that the plasma density has to be tapered from $\sim 2.5 \times 10^{19} \mathrm{~cm}^{-3}$ to $\sim 3 \times 10^{20} \mathrm{~cm}^{-3}$. Figure 3 (d) shows tha the density-gradient length defined by eq. (8) was larger than $150 \mu \mathrm{m}$.

The $v_{q} / c$ value required by eq. (8) is $\sim 0.03$, or laser intensity $I=3.90 \times 10^{15} \mathrm{~W} \cdot \mathrm{cm}^{-2}$. If we assume the waist size as $15 \mu \mathrm{m}$, the laser power becomes $27.5 \mathrm{GW}$. The laser pulse width of $15 \mathrm{ps}$ requires the laser energy of $413 \mathrm{~mJ}$. This laser intensity makes the tunneling ionization possible. Equation 7 tells that the $n_{1} / n_{0}$ value slightly exceeds unity in this laser intensity, though it does not take the spatial inhomogeneity into account.

\section{DISCUSSION}

There of course exist some problems to be solved in this method. First of all good simulation studies are necessary, which checks the phase continuity of the plasma wave in the density gradient, and estimates exact value of acceleration gradient and its time evolution.

The major technical problem is the creation of the plasma density gradient. A gas flow flux from an orifice limited by a skimmer has the radial distribution similar to that in Fig. 3(c)[5]. Further studies are necessary to apply this method to our experiment. Development of the chirped seed laser may become necessary to control the instability. Even if each technique were independently established, there would remain the problem of synchronization among the acceleration gradient, the plasma-density gradient and the laser-frequency chirping. Some good diagnostics and feedback control are essential to perform the experiment.

In conclusion, a method is proposed to accelerate protons by a slow plasma wave produced in the backward Raman scattering. It adjusts the plasma density to match the phase velocity of the plasma wave to the velocity of the protons accelerated. Energy of a commercially-available laser is large enough to excite such a plasma wave. Physical and technical problems are remain to be studied, which include Raman instability studies and realization of a tapered plasma.

One of the author (A.0.) is grateful to Dr. T. Ishida of Nagoya University for useful discussions on gas dynamics of a gas jet. The present work is supported by the JAERI's Nuclear Research Promotion Program.

\section{REFERENCES}

[1] A. Ogata and T. Katsouleas, Proc. 1998 Workshop on Advanced Accelerator Concepts, to be published, and references therein.

[2] T. Katsouleas et al., AIP Conf. Proc, vol.6, 63 (1985); N. A. Ebrahim and S. R. Douglas, Laser and Part. Beams, 13, 147 (1995).

[3] Advances in Plasma Physics, vol.6, P. K. Kaw et al., ed., John Wiley and Sons, New York (1976).

[4] J. M. Manley and H. E. Rowe, Proc. IRE, 44, 904 (1956); D. W. Forslund, J. M. Kindel and E. L. Lindman, Phys. Fluids, 18, 1002 (1975)

[5] J. B. Anderson, in Molecular Beams and Low Density Gas Dynamics, P.Wegener, ed., Marcel Decker, Ink., New York (1974), p5. 